\title{
Noise-induced transition from anomalous to ordinary diffusion: The crossover time as a function of noise intensity
}

\author{
Elena Floriani and Riccardo Mannella \\ Dipartimento di Fisica dell'Università di Pisa, Piazza Torricelli 2, 56100 Pisa, Italy \\ Paolo Grigolini \\ Dipartimento di Fisica dell'Università di Pisa, Piazza Torricelli 2, 56100 Pisa, Italy; \\ Department of Physics, University of North Texas, P.O. Box 5368, Denton, Texas 76203; \\ and Istituto di Biofisica del Consiglio Nazionale delle Ricerche, Via San Lorenzo 28, 56127 Pisa, Italy
}

(Received 10 July 1995)

\begin{abstract}
We study the interplay between a deterministic process of weak chaos, responsible for the anomalous diffusion of a variable $x$, and a white noise of intensity $\Xi$. The deterministic process of anomalous diffusion results from the correlated fluctuations of a statistical variable $\xi$ between two distinct values +1 and -1 , each of them characterized by the same waiting time distribution $\psi(t)$, given by $\psi(t) \simeq t^{-\mu}$ with $2<\mu<3$, in the long-time limit. We prove that under the influence of a weak white noise of intensity $\Xi$, the process of anomalous diffusion becomes normal at a time $t_{c}$ given by $t_{c} \sim 1 / \Xi^{\beta(\mu)}$. Here $\beta(\mu)$ is a function of $\mu$ which depends on the dynamical generator of the waiting-time distribution $\psi(t)$. We derive an explicit expression for $\beta(\mu)$ in the case of two dynamical systems, a one-dimensional superdiffusive map and the standard map in the accelerating state. The theoretical prediction is supported by numerical calculations.
\end{abstract}

PACS number(s): 05.40. $+\mathrm{j}, 05.60 .+\mathrm{w}$

\section{INTRODUCTION}

The deterministic approach to anomalous diffusion has been intensively studied in the last few years by several groups [1-3]. The map originally introduced by Geisel and co-workers [1] is now regarded [4] as the prototype for deterministic dynamics leading to anomalous diffusion faster than ordinary Brownian motion. It is a onedimensional map driving the motion of a variable, $\xi$, which can be thought of as the velocity of a diffusing particle whose position is given by the variable $x$ itself. In the continuous time limit we have

$$
\frac{d x}{d t}=\xi(t)
$$

and the map of Ref. [1] makes the variable $\xi$ fluctuate between the two states, $\xi=1$ and $\xi=-1$. The sojourn time in each state is characterized by the distribution $\psi(t)$, which has the inverse power-law structure

$$
\lim _{t \rightarrow \infty} \psi(t)=\frac{\text { const }}{t^{\mu}}
$$

in the long-time limit, with $\mu>1$. Here we focus our attention on the range

$$
2<\mu<3 .
$$

This choice is determined by two distinct but related reasons: (i) The range of Eq. (3) has been proved [2,3] to correspond to the dynamical realization of an $\alpha$ stable Lévy diffusion process. We remind the reader that Lévy processes of diffusion are $[5,6]$ a generalization of Brownian motion, characterized by distributions $\rho(x)$ whose Fourier transforms have the form

$$
\hat{\rho}(k)=e^{-|k|^{\alpha}},
$$

with the parameter $\alpha$ in the range $0<\alpha<2$. The deterministic approach of Refs. [2] and [3] connects $\alpha$ and $\mu$ via the relation $\alpha=\mu-1$. Thus, the region given by Eq. (3) corresponds to the parameter $\alpha$ ranging from $\alpha=2$ (Gaussian diffusion) to $\alpha=1$ (ballistic motion) and this range of the parameter has been shown to be compatible with an equilibrium dynamical realization of Lévy processes. Moreover, the recent investigations of many groups [4] suggest that Lévy processes are as ubiquitous as the Brownian motion itself. (ii) The theoretical investigations of Refs. $[2,3]$ have shown that the asymptotic properties of a process of anomalous diffusion are independent of the details of the dynamical generator of the distribution $\psi(t)$ and that they depend only on the time asymptotic property of (2). The standard map in the accelerating state is proved $[7,8]$ to result in a waitingtime distribution in each of the two accelerating modes with the same inverse power structure as that of (2) with the index $\mu$ fulfilling the condition (3). Thus, the anomalous diffusion generated by the standard map "coincides" with the one generated by the map of Geisel, Nierwetberg, and Zacherl [1] if the index $\mu$ is the same, and both are a dynamical realization of a Lévy process.

The main purpose of this paper is to study the transition from anomalous to normal diffusion triggered by environmental fluctuations. In the literature there are already investigations of this kind $[9,10]$ and the interest for this problem in our case is dictated by the following reasons. 
(a) As already pointed out in [9] the Hamiltonian dynamical realization of a process of anomalous diffusion in complex systems implies the interaction of the source of the inverse power-law distribution with a virtually infinitely large number of degrees of freedom. This leads to a transition from the inverse power-law behavior of (2) to an exponential decay. In Ref. [9] this transition was assessed numerically and the focus of the paper was on the dependence of the resulting diffusion coefficient on this crossover time with no discussion of how this crossover time depends on the intensity of the environment fluctuations. The purpose of this paper is to establish this dependence by means of a simple analytical expression.

(b) According to recent theoretical work [4], the roots of anomalous diffusion in Hamiltonian systems rest on the self-similar properties of the region at the border between the chaotic sea and the accelerating islands. Selfsimilarity with no lower bound is an idealization of reality conflicting with the quantum-mechanical nature of the physical systems. It has been recently assessed [11], on the basis of both theoretical and numerical arguments, that this implies a transition from anomalous to normal diffusion at a quantum crossover time $t_{\mathrm{QC}}$ that depends logarithmically on the Planck constant $\hbar$,

$$
t_{\mathrm{QC}} \sim \frac{1}{\lambda} \ln \frac{1}{\hbar}
$$

where $\lambda$ is the Lyapunov coefficient of the trajectories moving in the chaotic sea embedded within the fractal region at the border with the accelerating islands [4]. One would expect that for a macroscopic system environmental fluctuations are a cause of breakdown of anomalous diffusion more significant than quantum fluctuations. This would imply that the crossover time of the transition, caused by thermal fluctuations, depends logarithmically, or more weakly, on the intensity of the corresponding fluctuations. We shall show, on the contrary, that this crossover time, $t_{C}$, fulfills the relation

$$
t_{C} \sim \frac{1}{\Xi^{\beta(\mu)}}
$$

where $\beta(\mu)$ is a simple analytical function of $\mu$, which turns out to be of the order of unity.

The outline of the paper is as follows. Sec. II is devoted to applying our theoretical approach to the map of Geisel, Nierwetberg, and Zacherl [1]. In Sec. III the same theoretical approach is applied to the standard map. In Sec. IV these theoretical predictions are checked with the help of computer calculations. Finally, in Sec. V we point out the physical significance of the results obtained.

\section{THE ONE-DIMENSIONAL DYNAMICAL MODEL}

The discrete-time dynamical model studied here is one of the two discussed by Zumofen and Klafter [2], originally introduced by Geisel, Nierwetberg, and Zacherl [1]. We focus on the map they used to discuss anomalous diffusion that evolves faster than normal (superdiffusion).
Using the property of antisymmetry by reflection around $x=0$ and the invariance by translation of a unit cell, they express their map in the reduced range $0 \leq x \leq 1 / 2$ as

$$
x_{n+1}=g\left(x_{n}\right)
$$

with

$$
g(x)=x+a x^{z}-1,
$$

where the constant $a$ is given by $a=2^{z}$.

The reduced map, which is obtained iterating separately the motion within each unitary cell and the motion between cells, is defined as [2]

$$
\begin{aligned}
y_{n+1} & =g^{*}\left(y_{n}\right), \\
N_{n+1} & =N_{n}+\hat{g}\left(y_{n}\right),
\end{aligned}
$$

where $0 \leq y_{n} \leq 1$ and $x_{n}=y_{n}+N_{n}$ (see Fig. 1)

To simulate the interaction with the environment we perturb the deterministic dynamics of Eqs. (7), (8), and (9) adding a Gaussian white noise of (small) intensity $\Xi$, defined by

$$
\left\langle f_{i} f_{j}\right\rangle=2 \Xi \delta_{i, j}
$$

The map dynamics under the influence of environmental fluctuations thus becomes

$$
\begin{aligned}
y_{n+1} & =g^{*}\left(y_{n}\right)+f_{n}, \\
N_{n+1} & =N_{n}+\hat{g}\left(y_{n}\right) .
\end{aligned}
$$

In the original mapping the diffusion between cells could not take place through the boundaries (in the unitary cell) $y=0$ or $y=1$, but only via the map driving the cell number. In principle, the addition of noise opens up also the possibility of diffusion through the boundaries. To avoid this, which would imply a major perturbation of the original mapping, we introduced reflecting boundary conditions at $y=0$ and $y=1$ for the evolution of $y_{n}$, once the noise is switched on.

We are now in a position to realize a discrete-time rep-

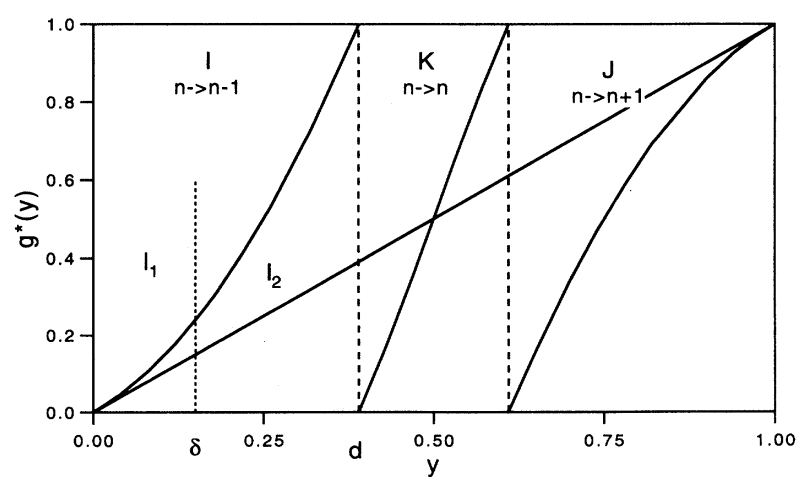

FIG. 1. The reduced map of Eq. (11). Note the regions $I$, $J$, and $K$, and the points $d$ and $\delta$. 
resentation of the generic equation of motion

$$
\dot{x}=\xi \text {. }
$$

The motion in the regions $I, J$ has a regular (laminar) character; the particle remains there for a large number of iterations. Permanence in region $I(J)$ corresponds to an increment $-1(+1)$ of the variable $x$ at each iteration. Regions $I$ and $J$ therefore correspond to the velocity $\xi$ having the value $-1,+1$, respectively. In the absence of noise, the waiting-time distribution in each of the two regions is given $[1,2]$ by the inverse power law of Eq. (2) with

$$
\mu=\frac{z}{z-1}
$$

The laminar motion is interrupted by short phases of chaotic motion, corresponding to iterations in the central region $K$. The two phases (laminar and chaotic) alternate intermittently.

Let us refer to the laminar region on the left of the unitary cell, i.e., the region $I$ of size $d$ defined by $0 \leq y \leq d$. Analogous considerations apply to the region $J$. Let us divide the left laminar region into the two intervals $I_{1}$ defined by $0 \leq y \leq \delta$ and $I_{2}$ defined by $\delta \leq y \leq d$. The quantity we are interested in is the distribution of sojourn times in the interval $I$, in the presence of noise. We shall solve the problem, making the following simplifying assumption: the interval $I_{1}$ corresponds to a region where the dynamics is dominated by diffusion, while the interval $I_{2}$ corresponds to a region where the dynamics is dominated by the deterministic motion (9). This is so because while the deterministic dynamics slows down as we approach $y=0$, the dynamics induced by the noise is independent of the point in $I$ we consider. The problem of a proper definition of $\delta$, i.e., the boundary between the interval $I_{1}$ and the interval $I_{2}$, will be tackled later.

Let us first show how to express the distribution of sojourn times $\psi(t)$ in the interval $I$ in terms of $\psi_{1}(t)$ and $\psi_{2}(t)$, the waiting-time distributions relative to the intervals $I_{1}$ and $I_{2}$, respectively. Let $\eta\left(y_{0}, t\right)$ be the distribution of sojourn times in the interval $I$ if the particle is located at $y_{0}$ at $t=0$. We also introduce $\eta_{1}\left(y_{0}, t\right)$ and $\eta_{2}\left(y_{0}, t\right)$, the distributions corresponding to the sojourn in $I_{1}$ and $I_{2}$ with $y_{0}$ belonging to $I_{1}$ and $I_{2}$, respectively.

If $y_{0}$ belongs to $I_{1}$, the particle will move within $I_{1}$ under the influence of diffusion, it will reach the border with $I_{2}$ and then it will move within $I_{2}$ driven by the deterministic motion. Notice that the motion in the laminar phase takes place only in one direction, from within the two laminar regions towards the central chaotic region. Thus, after entering the interval $I_{2}$, the particle moves towards the border $y=d$ with no recrossings, but those induced by noise, which, by hypothesis, are supposed to be negligible in the region $I_{2}$. If $y_{0}$ belongs to $I_{2}$, the only possible dynamics left to the particle is the deterministic motion towards the border $y=d$. Notice that adopting the continuous-time representation [1], we can evaluate the time $t(\delta)$ it takes the particle to move from $y=\delta$ to $y=d$. This reads

$$
t(\delta)=\frac{\delta^{1-z}-d^{1-z}}{a(z-1)} .
$$

As a consequence of these dynamical properties we have

$$
\begin{aligned}
& \eta\left(y_{0}, t\right)=\eta_{1}\left(y_{0}, t-t(\delta)\right) \quad \text { if } y_{0} \in I_{1} \\
& \eta\left(y_{0}, t\right)=\eta_{2}\left(y_{0}, t\right) \text { if } y_{0} \in I_{2} .
\end{aligned}
$$

This is equivalent to defining $\eta\left(y_{0}, t\right)$ as

$$
\begin{aligned}
\eta\left(y_{0}, t\right)= & \theta\left(\delta-y_{0}\right) \theta(t-t(\delta)) \eta_{1}\left(y_{0}, t-t(\delta)\right) \\
& +\theta\left(y_{0}-\delta\right) \theta(t(\delta)-t) \eta_{2}\left(y_{0}, t\right),
\end{aligned}
$$

where $\theta(x)$ is the usual step function.

Assuming, as is usually done [2], that the particles are uniformly injected in the laminar region, we obtain

$$
\begin{gathered}
\psi(t)=\frac{1}{d} \int_{0}^{d} \eta\left(y_{0}, t\right) d y_{0} \\
\psi_{1}(t)=\frac{1}{\delta} \int_{0}^{\delta} \eta_{1}\left(y_{0}, t\right) d y_{0} \\
\psi_{2}(t)=\frac{1}{d} \int_{0}^{d} \eta_{2}\left(y_{0}, t\right) d y_{0}
\end{gathered}
$$

This is equivalent to expressing $\psi(t)$ as follows:

$\psi(t)=\theta(t-t(\delta)) \frac{\delta}{d} \psi_{1}(t-t(\delta))+\theta(t(\delta)-t) \psi_{2}(t)$

Let us now address the problem of evaluating $\psi_{1}(t)$ (the same calculation has been carried out by Agmon and Weiss in Ref. [12]). First, we note that the probability of finding the particle at $y$ if it was initially placed at $y_{0}$, assuming that both $y$ and $y_{0}$ belong to $I_{1}$ and thus the motion from $y_{0}$ to $y$ is diffusional, can be evaluated solving the standard diffusion equation

$$
\frac{\partial p}{\partial t}=\Xi \frac{\partial^{2} p}{\partial y^{2}}=\Xi \frac{\partial^{2} p}{\partial y_{0}^{2}},
$$

with initial condition

$$
p\left(y, t=0 \mid y_{0}\right)=\delta\left(y-y_{0}\right) .
$$

It follows from the dynamics that we must impose a reflecting boundary condition at $y=0$. This means $\left.\frac{\partial p}{\partial y}\right|_{y=0}=\left.\frac{\partial p}{\partial y_{0}}\right|_{y_{0}=0}=0$. Then we assume that, once the border $y=\delta$ is reached, the particle is immediately injected into the interval $I_{2}$. Consequently we set at $y=\delta$ an absorbing boundary condition $p\left(y=\delta, t \mid y_{0}\right)=$ $p\left(y, t \mid y_{0}=\delta\right)=0$. Then we define

$$
f_{1}\left(y_{0}, t\right) \equiv \int_{0}^{\delta} p\left(y \mid t, y_{0}\right) d y
$$

namely, the probability that the coordinate $y$ is still in $I_{1}$ at the time $t$ given the initial condition $y_{0}$ at $t=0$ (with $y_{0} \in I_{1}$ ). We define also the probability that the coordinate $y$ is still in $I_{1}$ given the initial condition of being somewhere in $I_{1}$ at $t=0$, 


$$
f_{1}(t) \equiv \frac{1}{\delta} \int_{0}^{\delta} f_{1}\left(y_{0}, t\right) d y_{0}
$$

It is straightforward to show that these definitions imply

$$
\begin{array}{r}
\eta_{1}\left(y_{0}, t\right)=-\frac{\partial}{\partial t} f_{1}\left(y_{0}, t\right), \\
\psi_{1}(t)=-\frac{\partial}{\partial t} f_{1}(t) .
\end{array}
$$

The solution of (21), with a reflecting boundary condition at $y=0$ and an absorbing boundary condition at $y=\delta$, is $[12]$

$$
\begin{aligned}
p\left(y, t \mid y_{0}\right)= & \sum_{n=1}^{\infty} \frac{2}{\delta} \cos \left[\left(n+\frac{1}{2}\right) \frac{\pi y}{\delta}\right] \cos \left[\left(n+\frac{1}{2}\right) \frac{\pi y_{0}}{\delta}\right] \\
& \times \exp \left[-\frac{\pi^{2} \Xi}{\delta^{2}}\left(n+\frac{1}{2}\right)^{2} t\right] .
\end{aligned}
$$

Using (23) and (24), we derive

$$
f_{1}(t)=\sum_{n=0}^{\infty} \frac{2}{\delta^{2}} \frac{\exp \left[-\frac{\pi^{2} \Xi}{\delta^{2}}\left(n+\frac{1}{2}\right)^{2} t\right]}{\left(n+\frac{1}{2}\right)^{2}}
$$

Substituting (28) in (26) we get

$$
\psi_{1}(t)=\sum_{n=0}^{\infty} \frac{2 \Xi}{\pi^{2}} \exp \left[-\frac{\pi^{2} \Xi}{\delta^{2}}\left(n+\frac{1}{2}\right)^{2} t\right] .
$$

We note that this expression fulfills the normalization condition

$$
\int_{0}^{\infty} \psi_{1}(t) d t=1
$$

The calculation of $\psi_{2}(t)$ is carried out as in Ref. [1] and leads to

$$
\psi_{2}(t)=\frac{a}{d}\left[(z-1) a t+d^{1-z}\right]^{\frac{z}{1-z}}
$$

The total waiting-time distribution $\psi(t)$ of Eq. (20) is completely defined, since $\psi_{1}(t)$ is given by Eq. (29) and $\psi_{2}(t)$ by $\mathrm{Eq}(31)$. It is immediate to verify that the waiting-time distribution $\psi(t)$ is also correctly normalized.

Let us now approach the central problem of this section. We have to define the parameter $\delta$ as a function of $\Xi, a$, and $z$. The criterion adopted to define $\delta$ is the following: we define $\left\langle t_{1}\right\rangle$ as the mean sojourn time in $I_{1}$, evaluated with the assumption that the dynamics within $I_{1}$ is only due to diffusion; then we evaluate the mean sojourn time under the assumption that the dynamics of the system depends only on the deterministic motion of the unperturbed map (i.e., we fictitiously remove the noise), and we denote it by the symbol $T_{\delta}$. The parameter $\delta$ is determined by the condition

$$
\left\langle t_{1}\right\rangle \approx T_{\delta}
$$

Then, $\left\langle t_{1}\right\rangle$ can be determined using $\psi_{1}(t)$ from (29), and we have

$$
\left\langle t_{1}\right\rangle=\int_{0}^{\infty} t \psi_{1}(t) d t=\sum_{n=0}^{\infty} \frac{2 \delta^{2}}{\pi^{2} \Xi} \frac{1}{\left(n+\frac{1}{2}\right)^{2}}=\frac{\delta^{2}}{3 \Xi} .
$$

The determination of $T_{\delta}$ comes from

$$
T_{\delta}=\frac{1}{\delta} \int_{0}^{\delta} d y_{0} T\left(y_{0}\right)
$$

where $T\left(y_{0}\right)$ is the time it takes a deterministic trajectory driven by the unperturbed map to reach the border $\delta$ from the initial position. $T\left(y_{0}\right)$ turns out to be

$$
T\left(y_{0}\right)=\frac{y_{0}^{1-z}-\delta^{1-z}}{a(z-1)}
$$

so that we get the following result:

$$
T_{\delta}=\frac{\delta^{1-z}}{a(2-z)} .
$$

Using (36) and (33), we derive from the condition (32) the following estimate for $\delta$ :

$$
\delta^{1+z} \simeq \Xi
$$

Using (13) we derive from (37) the result

$$
\delta \simeq \Xi^{(\mu-1) /(2 \mu-1)} .
$$

We see from Eqs. (20) and (29) that the asymptotic behavior of $\psi(t)$ is given by

$$
\psi(t) \simeq \exp \left[-\left(\frac{\Xi}{\delta^{2}}\right) t\right]
$$

Using (38) we finally derive

$$
\psi(t) \simeq \exp \left[-\Xi^{1 /(2 \mu-1)} t\right]
$$

or, writing explicitly the crossover time,

$$
t_{c} \sim \Xi^{-1 /(2 \mu-1)} \text {. }
$$

Writing [see also Eq. (6)] the relation between $t_{c}$ and $\Xi$ in the form

$$
t_{c} \sim \frac{1}{\Xi^{\beta(\mu)}}
$$

for this model we have that

$$
\beta(\mu)=\frac{1}{2 \mu-1} .
$$

This prediction will be checked in Sec. IV with the help of numerical calculations. We have to stress, however, that this prediction is also supported by the renormalization-group arguments of Sec. III.

We would like, now, to establish a connection with the results of a preceding paper of our group [9]. In that paper it was proved that if the long-time behavior of $\psi(t)$, exhibiting at intermediate times an inverse power-law behavior like that of (2), is dominated by the exponential 
behavior $\psi(t) \sim \exp (-\epsilon t)$, then in the asymptotic time limit we will recover standard diffusion with a diffusion coefficient that is proportional to $\epsilon^{\mu-3}$. We want to prove that the theory developed in this section fits this result.

Let us note first [9] that in the long-time limit

$$
\left\langle x^{2}(t)\right\rangle=\frac{\left\langle t^{2}\right\rangle}{\langle t\rangle} t,
$$

where $\left\langle t^{n}\right\rangle$ is the $n$th moment of $\psi(t)$. Using (20) we find

$$
\begin{aligned}
\langle t\rangle & =\int_{0}^{t(\delta)} t \psi_{2}(t) d t+\frac{\delta}{d} \int_{0}^{\infty}\left[t^{\prime}+t(\delta)\right] \psi_{1}\left(t^{\prime}\right) d t^{\prime} \\
& =\int_{0}^{t(\delta)} t \psi_{2}(t) d t+\frac{\delta}{d}\left[\left\langle t_{1}\right\rangle+t(\delta)\right] .
\end{aligned}
$$

The second term vanishes for $\delta$ going to zero, whereas the first term is independent of $\delta$. Thus, for $\Xi \rightarrow 0$, we get

$$
\langle t\rangle=\frac{d^{1-z}}{a(2-z)} .
$$

As far as $\left\langle t^{2}\right\rangle$ is concerned, we get

$$
\begin{aligned}
\left\langle t^{2}\right\rangle= & \int_{0}^{t(\delta)} t^{2} \psi_{2}(t) d t \\
& +\frac{\delta}{d} \int_{0}^{\infty}\left[t^{\prime 2}+2 t(\delta) t^{\prime}+t^{2}(\delta)\right]^{2} \psi_{1}\left(t^{\prime}\right) d t^{\prime} \\
= & \int_{0}^{t(\delta)} t^{2} \psi_{2}(t) d t+\frac{\delta}{d}\left[\left\langle t_{1}^{2}\right\rangle+2 t(\delta)\left\langle t_{1}\right\rangle+t^{2}(\delta)\right]^{2}
\end{aligned}
$$

Both terms diverge for $\delta$ going to zero. The leading divergence is

$$
\lim _{\delta \rightarrow 0}\left\langle t^{2}\right\rangle \simeq \delta^{3-2 z}
$$

Thus we find from (45) and (38) that

$$
\left\langle x^{2}(t)\right\rangle \simeq \delta^{(\mu-3) /(\mu-1)} t \simeq \Xi^{(\mu-3) /(2 \mu-1)} t .
$$

Identifying $\epsilon$ with $\Xi^{1 /(2 \mu-1)}$ [see Eq. (40)] we recover the result of the preceding paper [9]. We remark that the transition from anomalous superdiffusion to normal diffusion results in an enhancement of the diffusion coefficient $\Xi^{(\mu-3) /(2 \mu-1)}$, which is proportional to an inverse power of the small noise intensity $\Xi$.

We note that the result (49) is consistent with the predictions of $[10]$. In fact, the physical situation discussed in that paper in the case where the map displays intermittent behavior is the same discussed here in the particular case $z=2(\mu=2)$. It is immediate to verify that the prediction for the diffusion coefficient given by Eq. (34) of Ref. [10] is the same of Eq. (49) with $\mu=2$.

At first sight, the two-state approximation used in this section seems too drastic to provide accurate predictions. However, we think that its description of the asymptotic properties is correct, as the recovery of the prediction of Refs. [9] and [10] seems to suggest. In Sec. III we shall give a firmer support to this view adopting the renormalization-group method.

\section{THE RENORMALIZATION-GROUP APPROACH AND THE STANDARD MAP IN THE ACCELERATING MODES}

Let us again consider the reduced map (9), focusing on the behavior near the tangent bifurcation it exhibits at $y=0$. The map reads

$$
y_{n+1}=f\left(y_{n}\right)
$$

with $f(y)$ defined by

$$
f(y)=y+a y^{z}
$$

We perturb it with a noise term as follows:

$$
f(y)=y+a y^{z}+\sqrt{2 \Xi} \zeta,
$$

where $\zeta$ is a white noise defined by

$$
\left\langle\zeta(t) \zeta\left(t^{\prime}\right)\right\rangle=\delta\left(t-t^{\prime}\right)
$$

It is known that the scaling properties of the intermittent transition from chaos to regular motion that takes place at a tangent bifurcation can be described by a renormalization-group ( $R G$ ) equation [13] (the original idea of a RG approach to the study of period-doubling bifurcations is due to Feigenbaum [14]). The scaling theory tells us that a characteristic time of the process $t(\Xi)$ rescales as

$$
t(\Xi) \sim \Xi^{\frac{\nu}{2}}
$$

where the exponent $\nu$ is calculated by solving and linearizing the corresponding renormalization-group equation. The analysis carried out in [13] gives

$$
\nu=-\frac{2(z-1)}{z+1}
$$

Using (13) we have

$$
t(\Xi) \sim \Xi^{-1 /(2 \mu-1)}
$$

If we interpret $t(\Xi)$ as the characteristic time scale for diffusion, or equivalently as the crossover time between power-law and exponential behavior of $\psi(t)$, then the relation given by (56) has the same functional dependence of Eq. (41), with $\beta(\mu)=1 /(2 \mu-1)$, which coincides with the one given by Eq. (43).

This is quite satisfactory and it encourages us to apply the two-state approach of Sec. II to the case of the standard map in its accelerating modes [6] where, on the other hand, the RG would not be applicable. From a formal point of view the standard map perturbed by the environmental fluctuations is given by 


$$
\begin{aligned}
& p_{n+1}=p_{n}+K \sin \theta_{n} \\
& \theta_{n+1}=\theta_{n}+p_{n+1}+f_{n}, \quad \bmod 2 \pi
\end{aligned}
$$

where $f_{n}$ is the white noise term defined in Eq. (10).

We approach the problem considering the model for transport in phase space proposed by Hanson, Cary, and Meiss [15]. We now recall the physical picture on which this model rests [16]. It is known that the phase space of a nonintegrable Hamiltonian system with two degrees of freedom consists of a mixture of regular components, in which the motion is quasiperiodic, and irregular components, in which the motion appears to be chaotic. Every island of stability in the chaotic sea is encircled by a set of cantori (cantori are the remnants of destroyed invariant tori, through which orbits must weave). The cantori that have a sufficiently irrational rotation number have a small flux across them and act as barriers to transport; they form a sequence converging to the boundary circle of the island, which is believed to always be a critical KAM torus. Critical tori exhibit scaling properties that also imply scaling for the fluxes across the encircling low-flux cantori.

In the model for transport developed in $[15,16]$ the transitions take place between states corresponding to the regions of phase space whose boundaries are two successive low-flux cantori. The motion of an orbit trapped in a state is regarded as random, and successive transitions are assumed to be uncorrelated. This means that the dynamics can be described by a Markov chain. The transition probability from the $i$ th to the $j$ th state is given by

$$
p_{i \rightarrow j}=\frac{\Delta W_{i, j}}{A_{i}}
$$

where $\Delta W_{i, j}$ is the flux through the cantorus connecting the $i$ th state to the $j$ th state. $A_{i}$ is the area of phase space accessible to the particle in the $i$ th state. This model rests on the scaling assumption

$$
\begin{aligned}
\Delta W_{i, i+1} & =\Delta W_{i+1, i}=\omega^{i} \Delta W_{0,1}, \\
A_{i} & =\left(\frac{\omega}{\epsilon}\right)^{i} A_{0}, \\
p_{i \rightarrow i+1} & =p_{0 \rightarrow 1} \epsilon^{i} .
\end{aligned}
$$

This approach leads to the waiting-time distribution of Eq. (2) with

$$
\mu=1+\frac{\ln \omega}{\ln \epsilon} .
$$

The speed of the deterministic motion becomes slower, increasing the index $i$ of the state considered, which is equivalent to the particle entering more and more deeply inside the fractal region at the border between the chaotic sea and the accelerating island. The role of the environment fluctuation given by the stochastic force appearing in (57) is to produce a slow diffusional motion all over the phase space available to the particle. However, there must exist a given integer $N$ such that the deterministic dynamics of the map for $i>N$ must become slower than the environment-induced diffusion. As in the case of the one-dimensional map of Sec. II, it is reasonable to expect that the resulting waiting-time distribution will behave as

$$
\psi(t) \simeq \exp \left(-\frac{t}{t_{N}}\right)
$$

where $t_{N}$ represents the crossover time between anomalous and normal diffusion. We can regard $t_{N}$ as the characteristic time at which trajectories that reach states $i>N$ give the dominant contribution to the total probability of permanence in the Markov chain. At the same time we notice that for small noise intensity, which implies $N \gg 1$, the crossover time is essentially identical to the transition time from the $(N-1)$ th to the $N$ th state. This implies that, due to the nature of the model adopted, $t_{N}$ rescales as

$$
t_{N} \sim \epsilon^{-N}
$$

On the other hand, from (60) we have that the phase space area relative to the state $N, A_{N}$, rescales as

$$
A_{N} \propto\left(\frac{\omega}{\epsilon}\right)^{N} \text {. }
$$

The dependence of $N$ on the noise intensity can be determined by noticing that the connection between $t_{N}$ and $A_{N}$ must be

$$
\Xi t_{N} \approx A_{N}
$$

i.e., physically, that the phase state $A_{N}$ is "filled" by the noise.

Using Eqs. (64) and (65), from Eq. (66) we get

$$
\Xi \approx \omega^{N} \text {. }
$$

Using Eqs. (64) and (67) we get

$$
t_{N} \sim \epsilon^{-(\ln \Xi / \ln \omega)}=\Xi^{-(\ln \epsilon / \ln \omega)}=\Xi^{-1 /(\mu-1)},
$$

which results in

$$
\psi(t) \simeq \exp \left[-\Xi^{1 /(\mu-1)} t\right]
$$

It is clear that Eq. (68) implies a relation between the cutoff time [here, $t_{N}$, see Eq. (63)] and the noise intensity $\Xi$ similar to Eq. (42), with the function $\beta(\mu)$ given by

$$
\beta(\mu)=\frac{1}{\mu-1} .
$$

It must be stressed that the relation (66) is equivalent to the criterion of Sec. II, based on the division of the laminar phase into two regions. The diffusion motion resulting from the environmental fluctuations alone does not have any time scale whereas the time it takes to cross a phase space region of size $A_{N}$ depends only on the fluctuation intensity $\Xi$. Thus Eq. (66) defines the site $N$ at which the importance of the diffusion motion becomes comparable to that of the deterministic process.

We believe that this is a reliable criterion. We think that the only element of weakness of our treatment is that it rests on the prediction of Eq. (62), which is based on the parameters $\omega$ and $\epsilon$, which are not rigorously derived 
from the Hamiltonian of the system. However, we can always look at $\omega$ and $\epsilon$ as effective parameters, taking into account the complex hierarchical structure of the real phase space. In practice, we can assume a $\mu$ value coinciding with that given by the numerical calculations. We shall see, in Sec. IV, that the numerical calculations lead indeed to results in satisfactory agreement with the theoretical prediction of Eq. (69).

\section{NUMERICAL RESULTS}

The first model we have studied numerically is the system given by Eq. (11), driven by a standard generator for the Gaussian noise. We typically followed 10000 trajectories, initially randomly distributed over the range $[0,1]$, for times up to $10^{5}$ iterations of the mapping. We focused on the interval $I$, and built the distribution of exit times from this region. Some typical $\psi(t)$ are shown in Fig. 2 as dots, for the same value of $\mu$ and different noise intensities.

We fitted the distribution of exit times with an exponential function (see the solid line in Fig. 2), and then we derived, via a power-law fit, the dependence of the crossover time on the noise intensity.

The comparison between the numerical results for the function $\beta(\mu)$ and the theory [Eq. (43)] is shown in Fig. 3, as a function of $\mu$ : we regard the agreement as excellent.

We turned next to the standard map of Eq. (57). The first problem here is to have a reliable value for the parameter $\mu$, which characterizes the universal behavior of the map itself: note that the only free parameter, beside the noise intensity, is $K$. To the best of our knowledge, there is no extensive work on this map, aiming at mapping out the dependence, if any, of $\mu$ on $K$ : thus, we limited ourselves to the numerical investigations of the map for a couple of well-known values of $K$, for which some phenomenology is already known [4]. For these values of $K$, the map is characterized by a chaotic motion over

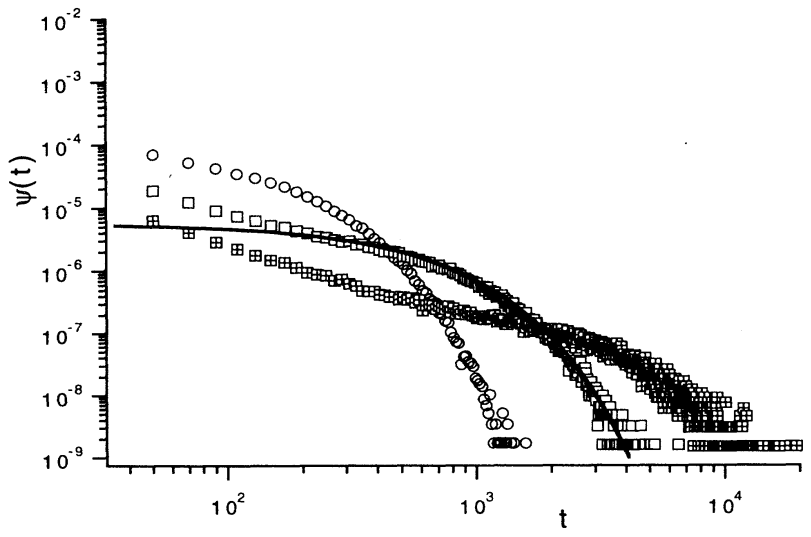

FIG. 2. Some typical $\psi(t)$ for the map of Eq. (11), for different noise intensities (symbols). The full line is the (asymptotic) best fit with the function $\exp \left(-t / t_{c}\right)$.

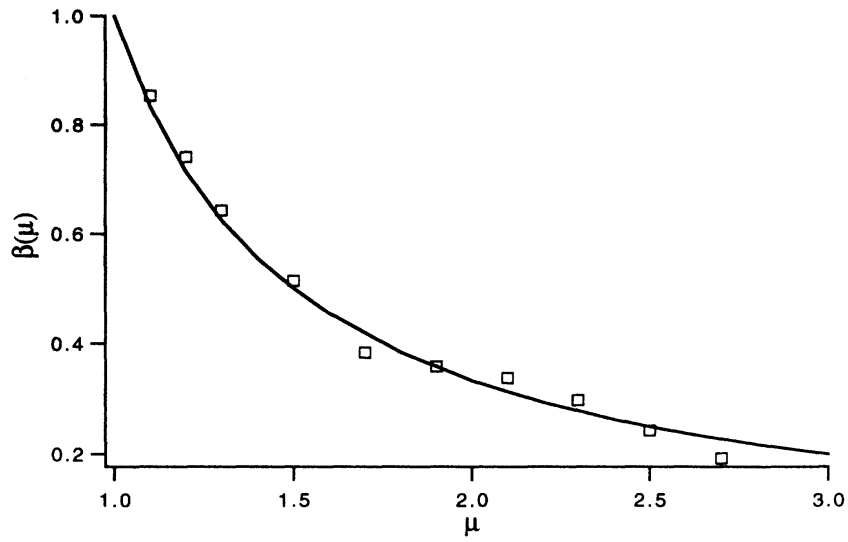

FIG. 3. Comparison between simulations (symbols) and theory (solid line) for the map of Eq. (11).

almost all the available phase space, apart from small regions (islands) of stable motion.

In this case it proved not very accurate to derive the crossover time directly: we decided then to look instead at the diffusion coefficient, which is directly connected to the crossover time [see Ref. [9] or the derivation leading to Eq. (49)]. For the sake of clarity, though, we will always express the numerical results in terms of $\beta(\mu)$.

We picked a number of initial starting points, making sure they were not within the periodic islands, and iterated them forward, computing the second moment of the variable $x$. Then, in the region where normal diffusion was established, we fitted a straight line to the second moment evolution, and derived a diffusion constant. Repeating the procedure for a number of different noise intensities we finally derived the dependence of the diffusion coefficient on the noise intensity $\Xi$. Finally, we "rescaled" the result in terms of a power expressing the dependence of $t_{c}$ on the noise intensity.

We found that for $K=6.91150$, for which $\mu=$ 2.667 [4], the simulations yield $\beta(\mu)=0.604$, and for $K=6.47168$, for which $\mu=2.302$ [4], the simulations yield $\beta(\mu)=0.8673$. Theoretically, from Eq. (70), we would expect $\beta(\mu)=0.600$ in the former case and $\beta(\mu)=0.7681$ in the latter case. We think that also for this system, although the dynamics is much more complex than in the previous case, the simulations agree well with the theory.

\section{CONCLUDING REMARKS}

In conclusion, we have shown that the crossover from anomalous to normal diffusion takes place at times $t_{c}$, which are inversely proportional to $\Xi^{\beta(\mu)}$,

$$
t_{c} \sim \frac{1}{\Xi^{\beta(\mu)}}
$$

with

$$
\beta(\mu)=\frac{1}{2 \mu-1}
$$


if the dynamical generator is the map of Eq. (8), and

$$
\beta(\mu)=\frac{1}{\mu-1}
$$

if the dynamical generator is the standard map in the presence of accelerating modes. This means that anomalous diffusion is fairly robust against the environmental fluctuations. Since $\Xi \ll 1$, the crossover time becomes longer and longer moving from $\mu=3$ (normal diffusion) to $\mu=2$ (ballistic motion). It must be noticed that in the standard case of $\xi$ characterized by an exponential relaxation function, there is no transition from anomalous to normal diffusion, and that the process of normal diffusion with a slightly increased diffusion coefficient is realized instantaneously. In the case of anomalous diffusion, on the contrary, as pointed out in [9], the diffusion coefficient of the process, after the transition from anomalous to normal diffusion took place, is anomalously large.

A further relevant aspect of this result is the sensitivity of the long-time regime of normal diffusion on the details of the dynamical generator. There is a general agreement on the universal nature of these processes: this means that different dynamical generators with the same waiting-time distribution lead to the same diffusion process. The effect of an environmental perturbation on an anomalous process of diffusion implies that the universal nature of the process is lost and that the crossover time (71) (and, consequently, the resulting diffusion coefficient [9]) depends on the nature of the dynamical generator, as is evident from the different dependence on $\mu$ of Eqs. (72) and (73). This may have interesting applications in distinguishing different microscopic dynamics leading to the same macroscopic behavior.

Finally, we must stress that the result of this paper might have remarkable consequences on the field of macroscopic effects of quantum mechanics. Numerical calculations [11] seem to support the conjecture that the crossover time from anomalous to normal diffusion caused by quantum fluctuation depends logarithmically on the strength of the fluctuations, i.e., the Planck constant, whereas, as shown in this paper, the crossover time of the corresponding process caused by environmental fluctuations is related to the intensity of these fluctuations, $\Xi$, by an inverse power-law relation [see Eqs. (72) and (73)]. This suggests that the transition to normal diffusion should be more sensitive to quantum than to thermal fluctuations. This is an interesting aspect requiring further investigation. It seems, however, that through the response to fluctuations it is possible to get information on the microscopic details of the dynamical generator, in spite of the universal character of the process and of the supposed independence of the macroscopic diffusion from microscopic dynamics.
[1] T. Geisel, J. Nierwetberg, and A. Zacherl, Phys. Rev. Lett. 54, 616 (1985).

[2] G. Zumofen and J. Klafter, Phys. Rev. E 47, 851 (1993).

[3] G. Trefan, E. Floriani, B. J. West, and P. Grigolini, Phys. Rev. E 50, 2564 (1994).

[4] J. Klafter, G. Zumofen, and M. F. Shlesinger (unpublished).

[5] A. Janicki and A. Weron, Simulation of Chaotic Behavior of $\alpha$-stable Stochastic Processes (Dekker, New York, 1994).

[6] E. W. Montroll and B. J. West, in Fluctuation Phenomena, edited by E. W. Montroll and J. L. Lebowitz (NorthHolland, Amsterdam, 1979).

[7] R. Ishizaki, H. Hata, T. Horita, and H. Mori, Prog. Theor. Phys. 84, 179 (1990); R. Ishizaki, T. Horita, T. Kobayashi, and H. Mori, ibid. 85, 1013 (1991).

[8] G. Zumofen and J. Klafter, Europhys. Lett. 25, 565
(1994).

[9] R. Bettin, R. Mannella, B. J. West, and P. Grigolini, Phys. Rev. E 51, 212 (1995).

[10] P. Reimann and Ch. Van den Broeck, Physica (Amsterdam) 75D, 509 (1994).

[11] R. Roncaglia, L. Bonci, B. J. West, and P. Grigolini, Phys. Rev. E 51, 5524 (1995).

[12] N. Agmon and G. H. Weiss, J. Phys. Chem. 93, 6884 (1989).

[13] J. E. Hirsch, M. Nauenberg, and J. Scalapino, Phys. Lett. 87 A, 391 (1982).

[14] M. Feigenbaum, J. Stat. Phys. 19, 25 (1978); 21, 669 (1979).

[15] J. D. Hanson, J. R. Cary, and J. D. Meiss, J. Stat. Phys. 39, 327 (1985).

[16] R. S. MacKay, J. D. Meiss, and I. C. Percival, Physica (Amsterdam) 13D, 55 (1984). 